\title{
Oscillations portuaires forcées par les ondes infra-gravitaires dans un chenal de navigation
}

\section{Florian BELLAFONT ${ }^{1}$, Denis MORICHON ${ }^{1}$, Volker ROEBER $^{2}$, Gaël ANDRÉ $^{3}$, Stéphane ABADIE ${ }^{1}$}

1. Université de Pau et des pays de l'Adour, Laboratoire SIAME - Fédération IPRA, EA4581, 64000, Pau, France.

bellafont.florian@univ-pau.fr denis.morichon@univ-pau.fr stephane.abadie@univpau.fr

2. Université de Hawaii à Manoa, Honolulu, Hawaii, États-Unis.

volker@hawaii.edu

3. SHOM, 13 rue du Chatellier, 29200 Brest, France.

gael.andre@shom.fr

\section{Résumé :}

Le port de Bayonne, situé proche de l'embouchure de l'Adour, rencontre fréquemment des problèmes de tenu à quai des navires en période de tempête. Afin de comprendre l'origine de l'agitation du port, une campagne de mesures, composée d'un houlographe mouillé à $50 \mathrm{~m}$ de fond et de trois capteurs de pression déployés dans le port, est réalisée durant un épisode de tempête de période de retour de 1 an $(\mathrm{Hs}=5 \mathrm{~m}$ et $\mathrm{Tp}=16 \mathrm{~s})$. Cet événement est également simulé avec un modèle numérique basé sur la résolution des équations de Boussinesq (BOSZ) afin de comprendre l'origine du forçage des oscillations mesurées dans le port. L'étude montre que les deux digues situées à l'embouchure de l'Adour assurent efficacement la protection du port contre la houle incidente en provoquant un amortissement de Hs de $85 \%$ dans la bande de périodes des vagues courtes (5-25 s). L'agitation du plan d'eau est majoritairement contrôlée par les ondes infra-gravitaires (IG). En période de tempête des valeurs de Hs de 0,70 m dont 0,65 $\mathrm{m}$ dans les IG (30-300 s) ont été mesurées à 1,4 km en amont du fleuve. Un dépôt sous-marin de dragage, situé entre 2,5 et 3,5 km des plages, provoque la levée des vagues et une augmentation de Hs de l'ordre de $20 \%$. Ce phénomène semble favoriser la génération d'ondes IG par interaction non linéaire entre les vagues courtes. En pénétrant dans le port, les ondes IG se propagent librement dans le fleuve et se dissipent progressivement par frottement. Cependant, elles conservent suffisamment d'énergie pour, d'une part, provoquer la résonance d'un port de plaisance situé à $1,4 \mathrm{~km}$ en amont de l'embouchure, et d'autre part probablement exciter les navires amarrés dont les périodes propres sont dans la bande IG.

Mots-clés :

Agitation portuaire, Tempêtes, Ondes infra-gravitaires, Modèle Boussinesq. 


\section{Thème 1 - Hydrodynamique côtière}

\section{Introduction}

L'embouchure d'un port est généralement dimensionnée pour protéger le bassin contre les vagues courtes gravitaires générées par le vent et dont la période est comprise entre 5 et $25 \mathrm{~s}$. Cependant, beaucoup de ports font face à des problèmes d'agitation du plan d'eau provoqués par les ondes longues ayant des périodes supérieures à $30 \mathrm{~s}$. Dans la plupart des cas, un port est une étendue d'eau semi-fermée située sur la côte et donc un système oscillant. Lorsque le forçage incident est suffisamment énergétique et que sa période est voisine de l'une des périodes propres du bassin, une onde stationnaire, associée à des oscillations verticales et des courants importants, se développe, on parle alors de seiche côtière (RABINOVICH, 2009).

Cependant, un régime stationnaire de seiche côtière peut difficilement s'établir dans les ports fluviaux, du fait qu'il n'y a pas de frontière amont sur laquelle peut se réfléchir une onde. Par contre, les ondes infra-gravitaires (IG), en pénétrant dans le fleuve, peuvent exciter le système constitué par le navire à quai et ses amarres (VAN DER MOLEN et al., 2006). Les ondes IG sont des ondes longues de surface de périodes comprises entre $30 \mathrm{~s}$ et $300-600 \mathrm{~s}$ (5-10 min) et générées par deux principaux mécanismes : l'interaction non linéaire entre les vagues courtes engendrant une onde IG liée aux groupes de vagues (LONGUET-HIGGINS \& STEWART, 1962), et la variation temporelle de la zone de déferlement agissant comme un batteur à onde longue (SYMONDS et al., 1982).

Le port en eau profonde de Bayonne (France), situé proche de l'embouchure de l'Adour, rencontre fréquemment des problèmes de tenu à quai des bateaux en période de tempête. Les objectifs de cette étude sont de caractériser l'agitation dans le port en période de tempête et de comprendre l'origine du forçage. Pour se faire, une campagne de mesures a été réalisée durant l'épisode du 3 février 2017 (Kurt) dont les conditions d'agitation au large sont typiques d'une tempête annuelle ( $\mathrm{Hs}=5 \mathrm{~m}$ et $\mathrm{Tp}=16 \mathrm{~s}$ ) (ABADIE et al., 2005). Dans un second temps, cet événement est simulé avec un modèle numérique de type Boussinesq afin de reproduire la propagation de la houle incidente du large vers la côte tout en prenant en compte l'effet de la bathymétrie sur la transformation des vagues et les processus à l'origine de la génération des ondes IG.

\section{Site d'étude}

Le port de Bayonne, localisé sur la façade sud-ouest de la France, s'étend jusqu'à 6,5 $\mathrm{km}$ en amont du fleuve de l'Adour. Sa profondeur moyenne est de $14 \mathrm{~m}$ par rapport au zéro hydrographique $(\mathrm{mCM})$. Le régime de marée est mésotidal. Les débits annuels minimum, moyen et maximum du fleuve sont respectivement de 30,300 et $2000 \mathrm{~m} / \mathrm{s}$. Deux digues protègent l'entrée du port contre les courants latéraux et la houle. Un dépôt sous-marin de sédiments issus des multiples dragages du fleuve est situé entre 2,5 et 3,5 $\mathrm{km}$ des plages et culmine à $-17 \mathrm{mCM}$. Un port de plaisance (port du Brise-Lames) d'une profondeur moyenne de 2,5 m est situé sur la rive gauche à 1,4 km de l'embouchure. 


\section{XVèmes Journées Nationales Génie Côtier - Génie Civil \\ La Rochelle, 29 au 31 mai 2018}

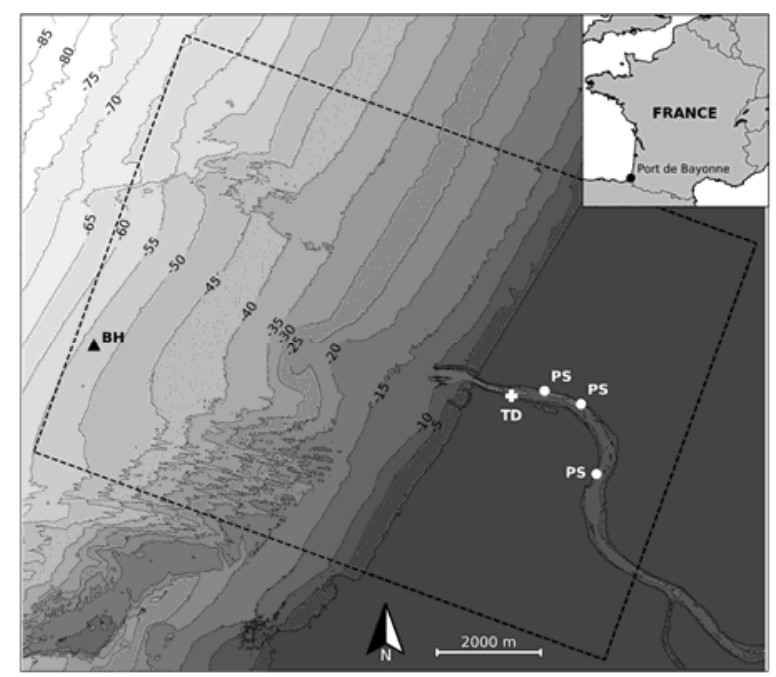

Figure 1. Port de Bayonne et positions des dispositifs expérimentaux : BH bouée de houle, TD marégraphe et PS capteur de pression. Traits pointillés : domaine du modèle.

\section{Données et méthodes}

Une bouée de houle, mouillée à $7 \mathrm{~km}$ de l'embouchure à une profondeur de $50 \mathrm{~m}$, mesure les conditions d'agitation au large toutes les $30 \mathrm{~min}$ et fournit un spectre de densité d'énergie intégré sur la direction. Le marégraphe Bayonne-Boucau situé à 850 $m$ de l'embouchure de l'Adour enregistre le niveau d'eau toutes les minutes. Le débit de l'Adour est obtenu à partir des données de la banque hydro (hydro.eaufrance.fr) et la pression atmosphérique est mesurée par une station météo située à Biarritz (données horaires). A l'approche de la tempête Kurt, trois capteurs de pression configurés pour une acquisition des données à $1 \mathrm{~Hz}$ ont été installés dans l'Adour à 1,4;2,2 et 3,7 km de l'embouchure. Les signaux de pression sont transformés en niveaux de surface libre en appliquant la formule de la pression hydrostatique dont le domaine d'application est respecté, puisque les capteurs sont situés à l'extérieur de la zone de déferlement et installés proche de la surface libre (profondeur de -1,5 $\mathrm{mCM}$ ).

Après avoir retirée la contribution de la marée en considérant une déviation linéaire du signal, la hauteur significative (Hs) est calculée à partir de séries de 2048 données de hauteur d'eau ( 34 min) selon la formule (1) (HOLTHUIJSEN, 2010).

$H s=4 \sqrt{m_{0}}$

avec Hs la hauteur significative et mo le moment d'ordre zéro.

Le choix des bornes d'intégration de mo permet de cibler les bandes de périodes des vagues courtes (5-25 s) et des ondes IG (30-300 s). Le spectre de densité d'énergie de la surface libre est déterminé en divisant le signal en segments de 2048 données avec un recouvrement de $50 \%$ entre chaque segment et en appliquant une fenêtre de Hanning. Le spectre moyenné étant relié à la variance de la surface libre, l'intervalle de confiance (IC) associé suit une loi du khi-deux $\left(\chi^{2}\right)$ de degré de liberté $v$ égal au nombre de segment moins 1 (formule 2) (THOMSON \& EMERY, 2014). 


\section{Thème 1 - Hydrodynamique côtière}

$I C=\left[\frac{v \cdot s^{2}}{\chi_{\alpha / 2, v}^{2}} ; \frac{v \cdot s^{2}}{\chi_{1-\alpha / 2, v}^{2}}\right]$

avec $v$ le degré de liberté, $\mathrm{s}^{2}$ la variance de l'échantillon, $\alpha$ le risque d'incertitude du spectre pris à $5 \%$.

\section{Modèle numérique}

La propagation des vagues depuis la bouée de houle jusque dans l'Adour pendant la tempête Kurt est simulée avec le modèle numérique BOSZ (ROEBER et al., 2010). Il s'agit d'un modèle de type Boussinesq basé sur les équations de NWOGU (1993) exprimées sous forme conservative. Dans le cas d'étude simulé, les effets du débit du fleuve et de la marée sont négligés. Pour respecter cette hypothèse, l'étude numérique est concentrée sur l'événement du 3 février 2017 de $8 \mathrm{~h}$ à $8 \mathrm{~h} 30$ (UTC) survenu à marée haute : le niveau d'eau était de 4,16 mCM en moyenne avec un écart-type de 0,03 m, et le débit de l'Adour était inférieur au débit moyen annuel $\left(190 \mathrm{~m}^{3} / \mathrm{s}\right.$ avec un écart-type de $8,5 \mathrm{~m}^{3} / \mathrm{s}$ ). Les caractéristiques des vagues mesurées au large étaient $\mathrm{Hs}=4,7 \mathrm{~m}, \mathrm{Tp}=18 \mathrm{~s}$, Dir au pic $=291^{\circ}$ avec un étalement directionnel au pic Etal $=17^{\circ}$. Le modèle BOSZ est forcé avec un spectre en fréquence-direction reconstitué à partir des mesures de la bouée de houle en utilisant une fonction d'étalement directionnel paramétrique (SWAN TEAM, 2007).

Le domaine de calcul (figure 1) est composé de mailles carrées de 6,5 $\mathrm{m}$ de côté, soit environ 2,4 millions de points dont $67 \%$ humides. Une rotation de $20^{\circ}$ du domaine dans le sens horaire permet d'orienter perpendiculairement la frontière ouest à la direction moyenne du forçage. Une condition éponge est appliquée aux frontières du domaine. Le frottement sur le fond est pris en compte à travers un coefficient de Manning pris égal à $0,015 \mathrm{~s} / \mathrm{m}^{3}$ sur tout le domaine, sauf au niveau des digues où un facteur 10 est appliqué pour tenir compte de l'effet des ouvrages sur la dissipation des vagues courtes.

La durée de la simulation est de 2 heures dont les 30 premières minutes permettent d'initialiser le modèle. Les 1 h30 restantes sont découpées en tronçon de 30 min dont les spectres de densité d'énergie sont moyennés. L'événement sélectionné est donc simulé trois fois. En effet, aucune information sur la phase n'est fournie dans le spectre de forçage du modèle. Pour combler ce manque, BOSZ assigne une phase de manière aléatoire à chaque fréquence du spectre et donc plusieurs simulations sont nécessaires pour reproduire correctement le spectre d'entrée.

\section{Résultats}

\subsection{Analyse des mesures}

L'événement étudié s'étend du 2 au 4 février 2017. Pendant cette période, la houle au large a atteint des valeurs maximales de $\mathrm{Hs}$ et Tp respectivement de $6 \mathrm{~m}$ et $18 \mathrm{~s}$ (figure 2 (a)). La figure 2 (b) montre la réponse du port au forçage en terme de Hs à 1,4 km de 


\section{XVèmes Journées Nationales Génie Côtier - Génie Civil \\ La Rochelle, 29 au 31 mai 2018}

l'embouchure. La contribution des vagues courtes dans l'agitation portuaire est faible $(\mathrm{Hssw}<0,20 \mathrm{~m})$ contrairement à celle des ondes IG dont les hauteurs significatives HsIG atteignent des valeurs de $0,65 \mathrm{~m}$ pour un $\mathrm{Hs}$ de $0,70 \mathrm{~m}$.
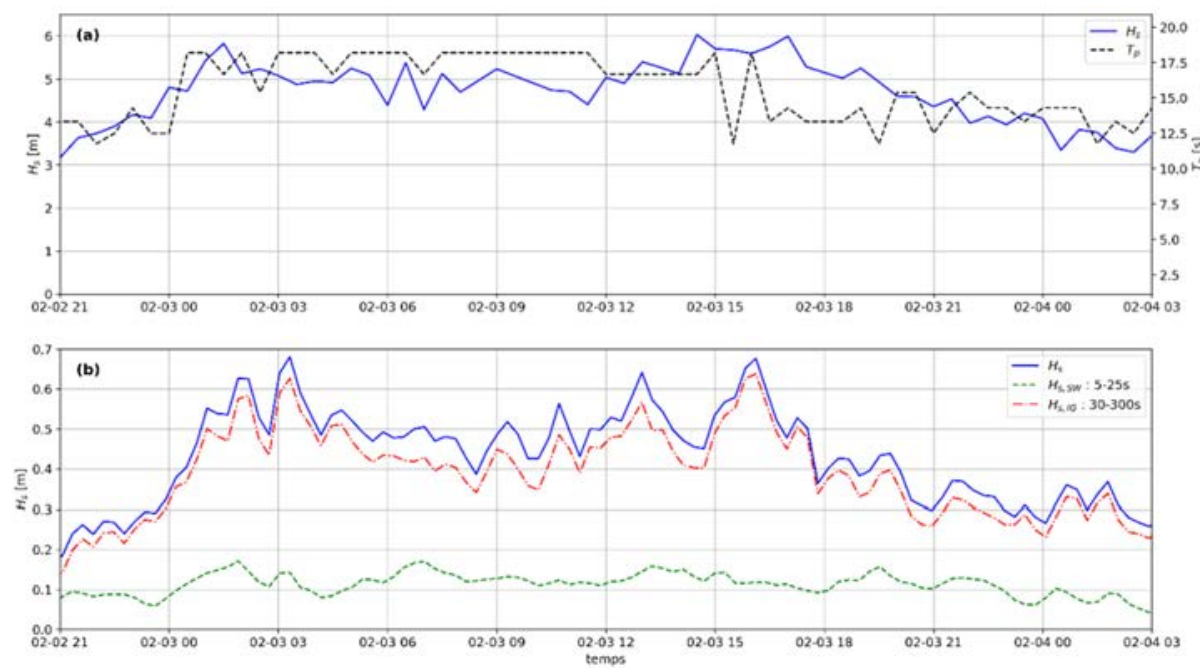

Figure 2. Evènement de tempête : (a) Bouée de houle et (b) Capteur aval (1,4km).

La figure 3 permet d'étudier la transformation des ondes lors de leur propagation dans le fleuve. Elle présente les spectres de densité d'énergie aux trois points de mesure, déterminés lorsque la valeur de Hs à $1,4 \mathrm{~km}$ de l'embouchure est supérieure à $0,40 \mathrm{~m}$ ( $\sim 17 \mathrm{~h}$ de données). On remarque que l'énergie diminue progressivement lors de la propagation des ondes dans le fleuve. L'énergie dans la bande des vagues courtes est très faible et se dissipe rapidement. Au contraire, l'énergie dans la bande IG est très importante et se dissipe peu (34\% de dissipation entre les points aval et amont).

Dans la bande de périodes des IG, deux bandes se distinguent : IG1 (30-120 s) et IG2 (120-300 s). La bande IG1, plus énergétique à 1,4 km de l'embouchure, perd plus d'énergie lors de la propagation dans le fleuve, si bien qu'à 3,7 km de l'embouchure la bande IG2 est majoritairement responsable de l'agitation du plan d'eau.

\subsection{Validation du modèle}

La figure 4 présente la comparaison entre les spectres de densité d'énergie déterminés à partir des niveaux d'eau mesurés et ceux simulés avec le modèle BOSZ, pour les trois points de mesure. La variation spatiale et la répartition fréquentielle de l'énergie sont bien reproduites par le modèle. Par ailleurs, la sous-estimation de l'énergie des ondes de périodes inférieures à $10 \mathrm{~s}$ est due à l'effet du vent local non pris en compte dans le modèle. Les comparaisons des spectres dans la bande IG montrent que le modèle BOSZ permet de reproduire la génération des IG par un forçage océanique et leur transformation au cours de la propagation dans le fleuve (tableau 2). 


\section{Thème 1 - Hydrodynamique côtière}

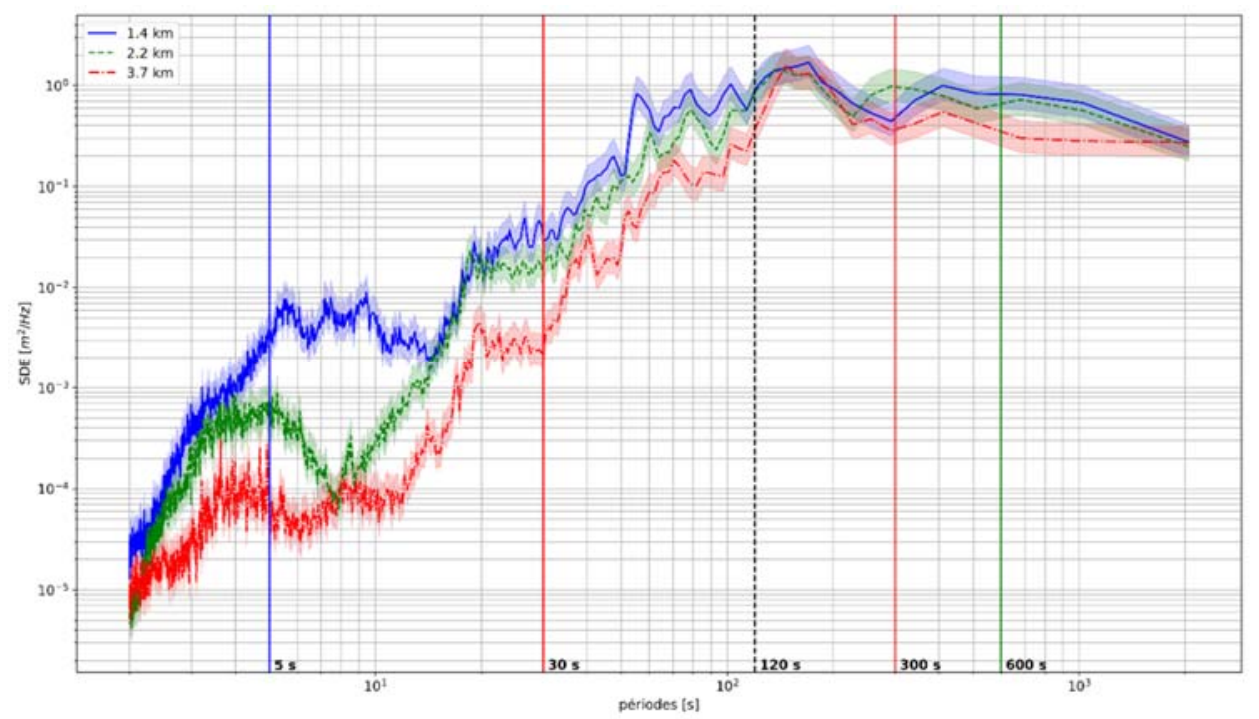

Figure 1. Spectres de densité d'énergie (SDE) et intervalles de confiance associés. Degré de liberté=59 et $\alpha=5 \%$.

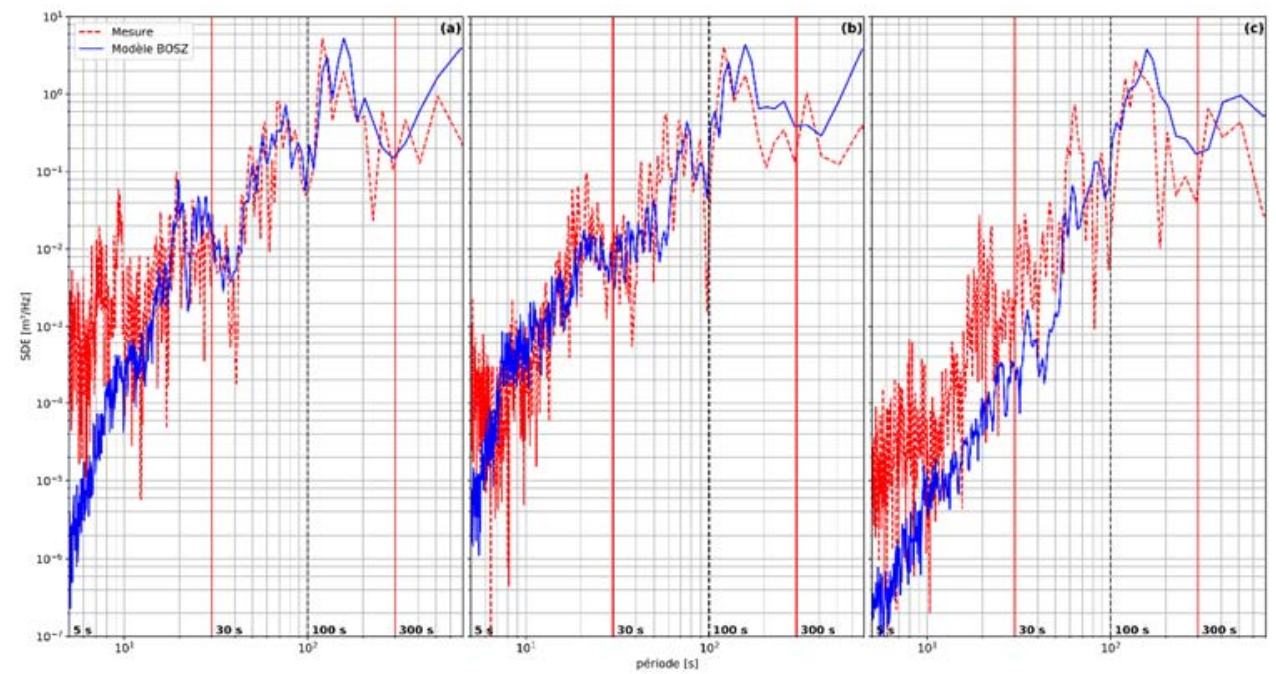

Figure 2. SDE aux points expérimentaux (courbes rouges) et numériques (courbes bleues) : (a) 1,4 km, (b) 2,2 km et (c) 3,7 km.

Tableau 2. Hs calculées à partir des spectres de densité d'énergie.

\begin{tabular}{|c|c|c|c|c|c|c|c|c|c|c|}
\hline & \multicolumn{3}{|l|}{1,4 km } & \multicolumn{3}{|l|}{$2,2 \mathrm{~km}$} & \multicolumn{3}{|l|}{$3,7 \mathrm{~km}$} \\
\hline & & Mesure & BOSZ & Écart & Mesure & BOSZ & Écart & Mesure & BOSZ & Écart \\
\hline & Périodes & $\mathrm{m}$ & $\mathrm{m}$ & $\%$ & $\mathrm{~m}$ & $\mathrm{~m}$ & $\%$ & $\mathrm{~m}$ & $\mathrm{~m}$ & $\%$ \\
\hline $\mathrm{Hs}$ & - & 0,44 & 0,46 & 5 & 0,41 & 0,43 & 5 & 0,35 & 0,37 & 6 \\
\hline SW & $5-25 \mathrm{~s}$ & 0,13 & 0,10 & - & 0,10 & 0,07 & - & 0,04 & 0,01 & - \\
\hline $\mathrm{IG}$ & $30-300 \mathrm{~s}$ & 0,40 & 0,44 & 10 & 0,38 & 0,41 & 8 & 0,33 & 0,35 & 6 \\
\hline IG1 & $30-100 \mathrm{~s}$ & 0,21 & 0,20 & - & 0,19 & 0,15 & - & 0,16 & 0,09 & - \\
\hline IG2 & $100-300 \mathrm{~s}$ & 0,34 & 0,39 & - & 0,33 & 0,38 & - & 0,28 & 0,34 & - \\
\hline
\end{tabular}




\section{XVèmes Journées Nationales Génie Côtier - Génie Civil \\ La Rochelle, 29 au 31 mai 2018}

\subsection{Mécanisme de générations des IG}

La figure 5 présente les $\mathrm{Hs}$ et Hsig calculées à partir des données de surface libre fournies par le modèle, avec un zoom sur la zone proche de l'embouchure. Au niveau de l'embouchure du fleuve, les valeurs de $\mathrm{Hs}$ diminuent de manière importante: atténuation de l'ordre de $85 \%$ entre le musoir de la digue nord et l'entrée du port. Au niveau du dépôt de dragage, les valeurs de Hs augmentent de l'ordre de $20 \%$ après son passage. La diminution des Hs à l'approche de la côte est provoquée par le déferlement des vagues. Globalement, les valeurs de $\mathrm{H}_{\text {sIG }}$ augmentent du large vers la côte et plus particulièrement au niveau du dépôt de dragage. Au niveau de l'embouchure du fleuve, les valeurs de Hsig diminuent de manière progressive lors de la pénétration des ondes dans le fleuve. On remarque un schéma typique de seiche dans le port de plaisance: oscillations maximales localisées aux frontières du bassin (anti-nœud de l'onde stationnaire) associées à des oscillations faibles voire nulles (nœud). Ce résultat confirme une étude précédente (PONS et al., 2008) qui a montré que les périodes propres du port de plaisance sont comprises dans la bande IG.
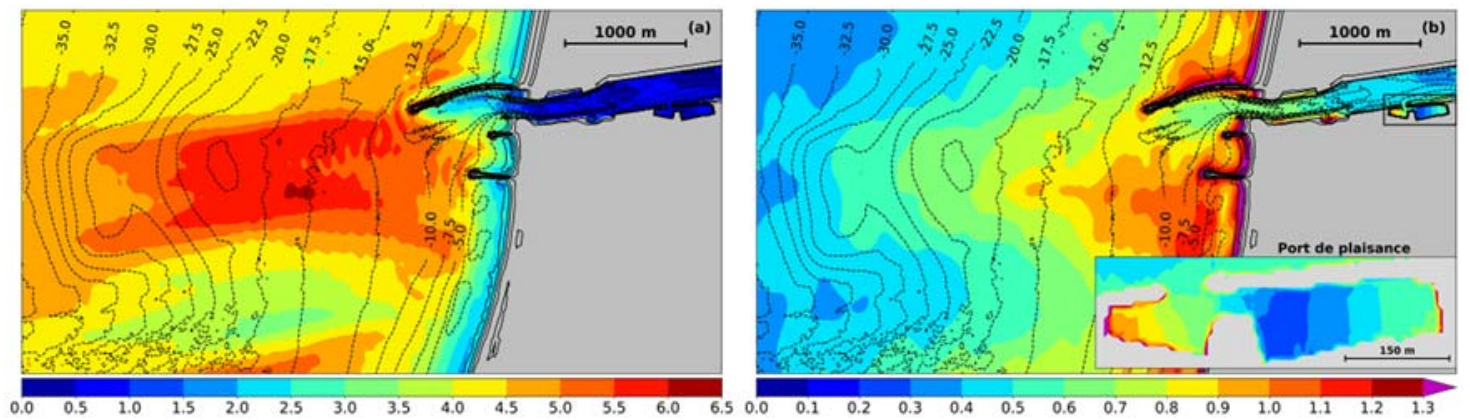

Figure 3. Carte de Hs (m) (a) et de Hsig (m) (b) issues du modèle. Isobathes (mCM) en traits pointillés noirs. Forçage : Hs=4,7 $\mathrm{m}$ et $\mathrm{Tp}=18 \mathrm{~s}$.

\section{Conclusion}

Cette étude basée sur des mesures de niveau d'eau et la simulation numérique de la propagation des vagues pendant un événement climatique énergétique montre que la contribution des vagues courtes (5-25 s) dans l'agitation du port de Bayonne est faible $(\mathrm{Hssw}<0,20 \mathrm{~m})$. Les deux digues situées à l'embouchure assurent efficacement la protection du port contre la houle incidente en provoquant un amortissement de Hs de $85 \%$. Les oscillations portuaires sont majoritairement contrôlées par les ondes IG (30300 s) qui sont générées par le forçage océanique (houle incidente). Le dépôt de dragage, situé au large de l'embouchure, semble participer à la génération d'ondes IG en favorisant l'interaction non linéaire entre les vagues courtes. En pénétrant dans le port, les ondes IG se propagent librement dans le fleuve qui fait office de guide d'onde et se dissipent progressivement par frottement. Cependant, elles conservent 


\section{Thème 1 - Hydrodynamique côtière}

suffisamment d'énergie, d'une part pour provoquer la résonance du port de plaisance du Brise-Lames, et d'autre part pour probablement exciter le navire amarré dont les périodes propres sont dans la bande IG.

\section{Références bibliographiques}

ABADIE S., BUTEL R., DUPUIS H., BRIÈRE C. (2005). Paramètres statistiques de la houle au large de la côte sud-aquitaine. Revue Comptes Rendus Geoscience, Vol. 337(8), pp 769-776. https://doi.org/10.1016/j.crte.2005.03.012

HOLTHUIJSEN L.H. (2010). Waves in oceanic and coastal waters. Cambridge university press.

LONGUET-HIGGINS, M.S., STEWART R.W. (1962). Radiation stress and mass transport in gravity waves, with application to "surf beats". Journal of Fluid Mechanics, Vol. 13(4), pp 481-504. https://doi.org/10.1017/S0022112062000877

NWOGU O. (1993). Alternative form of Boussinesq equations for nearshore wave propagation. Journal of waterway, port, coastal, and ocean engineering, Vol. 119(6), pp 618-638. https://doi.org/10.1061/(ASCE)0733-950X(1993)119:6(618)

PONS F., SERGENT P., LAMBERT A. (2008). Calcul des seiches à partir du logiciel Refonde. $\mathrm{X}^{\mathrm{èmes}}$ Journées Nationales Génie Côtier-Génie Civil, pp 14-16. https://doi.org/10.5150/jngcgc.2008.074-P

RABINOVICH A.B. (2009) Seiches and Harbor Oscillations. Chapter 9, Handbook of Coastal and Ocean Engineering. https://doi.org/10.1142/9789812819307_0009

ROEBER V., CHEUNG K.F., KOBAYASHI M.H. (2010). Shock-capturing Boussinesq-type model for nearshore wave processes. Coastal Engineering, Vol. 57(4), pp 407-423. https://doi.org/10.1016/j.coastaleng.2009.11.007

SWAN TEAM (2007). SWAN user manual. Delft University of Technology. The Netherlands.

SYMONDS G., HUNTLEY, D.A., BOWEN A.J. (1982). Two-dimensional surf beat: Long wave generation by a time-varying breakpoint. Journal of Geophysical Research: Oceans, Vol. 87(C1), pp. 492-498. https://doi.org/10.1029/JC087iC01p00492

THOMSON R.E., EMERY W.J. (2014). Data analysis methods in physical oceanography. Newnes.

VAN DER MOLEN W., MONARDEZ P., VAN DONGEREN A.P. (2006). Numerical simulation of long-period waves and ship motions in Tomakomai Port, Japan. Coastal Engineering Journal, Vol. 48(1), pp 59-79. https://doi.org/10.1142/S0578563406001301 